\title{
Magnetospheric ULF wave studies in the frame of Swarm mission: a time-frequency analysis tool for automated detection of pulsations in magnetic and electric field observations
}

\author{
Georgios Balasis $^{1}$, Ioannis A. Daglis ${ }^{2,1}$, Marina Georgiou ${ }^{1,2}$, Constantinos Papadimitriou ${ }^{1,2}$, and Roger Haagmans ${ }^{3}$ \\ ${ }^{1}$ Institute for Astronomy, Astrophysics, Space Applications and Remote Sensing, National Observatory of Athens, \\ Metaxa and Vasileos Pavlou, Penteli 15236 Athens, Greece \\ ${ }^{2}$ Section of Astrophysics, Astronomy and Mechanics, Department of Physics, University of Athens, \\ Panepistimioupoli Zografou, 15784 Athens, Greece \\ ${ }^{3}$ European Space Research and Technology Centre, European Space Agency, Noordwijk, The Netherlands
}

(Received March 15, 2013; Revised October 24, 2013; Accepted October 25, 2013; Online published November 22, 2013)

\begin{abstract}
We combine the advantages of multi-spacecraft and ground-based monitoring of the geospace environment in order to analyze and study magnetospheric ultra low frequency (ULF) waves. In line with this aim, we also develop and deliver relevant analysis tools based on wavelet transforms and tailored to the Swarm mission. In the preparation phase as well as the lifetime of the Swarm mission, the analysis of isolated ULF wave eventsespecially those detected in the Pc3 frequency range $(20-100 \mathrm{mHz})$ that a topside ionosphere mission efficiently resolves - can help to elucidate the processes that play a crucial role in the generation of waves and their most defining propagation characteristics. Additionally, we offer a useful platform to monitor the wave evolution from the outer boundaries of Earth's magnetosphere through the topside ionosphere down to the surface. Data from a single Low Earth Orbit (LEO) satellite (CHAMP), a multi-satellite LEO mission (ST5) and the ongoing multisatellite magnetospheric mission (Cluster) along with a ground-based magnetic network (CARISMA) are used to demonstrate the potential of our analysis technique in studying wave evolution in detail. A better understanding of the generation and propagation of waves will also allow to geophysically validate some of Swarm's data products, especially those related to the magnetic and electric fields in geospace. With a carefully selected case study focusing on the recovery phase of a moderate magnetic storm (9 April 2006 with a minimum $D_{\text {st }}$ value of $-82 \mathrm{nT}$ ) as a starting point, we clearly demonstrate the capabilities offered by our wavelet analysis tools and highlight the options opened to treat various categories of multipoint multi-instrument measurements (both spaceborne and ground-based) for signatures of ULF wave signals as well as the effects of various other sources. Key words: Magnetospheric ULF waves, LEO satellites, wavelet transforms.
\end{abstract}

\section{Introduction}

Ultra low frequency (ULF) waves are frequently observed throughout the Earth's magnetosphere as well as on the ground. Nonetheless, their source or sources, propagation characteristics and their implications to magnetosphere dynamics are still under debate. The broader class of ULF waves are quasi-sinusoidal and exist for several periods; these are classified as continuous and are further broken down into five subcategories, Pc1-5, depending upon their frequency (Jacobs et al., 1964). Their energy source is either in the solar wind or within the magnetosphere. Internally generated ULF waves may draw their energy from drift mirror instabilities due to pressure anisotropies (Cheng and Lin, 1987) and low frequency instabilities of the ring current plasma (Chen and Hasegawa, 1991), while externally generated from processes such as shear instabilities at the magnetopause flanks or compressive fluctuations of the solar wind (Menk, 2011 and references therein). On the

Copyright (c) The Society of Geomagnetism and Earth, Planetary and Space Sciences (SGEPSS); The Seismological Society of Japan; The Volcanological Society of Japan; The Geodetic Society of Japan; The Japanese Society for Planetary Sciences; TERRAPUB.

doi:10.5047/eps.2013.10.003 other hand, magnetospheric ULF waves have a profound effect on the dynamics of radiation belts (Brautigam and Albert, 2000; Ozeke et al., 2012 and the review by Elkington, 2006). As radiation belts variability has a direct impact on spacecraft and humans in space, ULF waves are of particular importance for space weather.

ULF waves are large-scale phenomena both in a spatial and a temporal manner. Thus, simultaneous observations at different locations and over long time intervals are needed to fully understand their generation (Takahashi and Anderson, 1992). Recent multi-satellite magnetospheric missions, such as ESA's Cluster and NASA's THEMIS, have opened the way for significant advances in the study of ULF waves (e.g. Constantinescu et al., 2007; Usanova et al., 2008; Sarris et al., 2009; Pickett et al., 2010). It is, nonetheless, anticipated that the upcoming ESA's topside ionosphere multi-satellite Swarm mission (Friis-Christensen et al., 2006) will bridge the gap between multipoint ULF wave observations made independently in the magnetosphere by missions such as Cluster and THEMIS and on the Earth's surface by ground-based networks of magnetic stations. It is expected to shed light on the propagation of ULF waves from the outer magnetosphere, through the ionosphere and 
down to Earth.

In particular, measurements of the geomagnetic field by LEO satellites, such as the CHAMP and ST5 satellites, have revealed ULF waves in the topside ionosphere (see Sutcliffe and Lühr, 2003; Balasis et al., 2005; Heilig et al., 2007; Le et al., 2011 and the review by Pilipenko et al., 2011). Pc3 waves have been clearly seen in the field-aligned (compressional) component of the magnetic field simultaneously with Pc3 waves observed on the ground (Heilig et al., 2007). Furthermore, $\mathrm{Pi} 2$ waves have been observed in the nightside magnetosphere at relatively low latitude (Sutcliffe and Lühr, 2010). The compressional component of these waves mapped directly to variations of the magnetic field $\mathrm{H}$ component of the measured on the ground (Han et al., 2004). Interestingly, the compressional component was in phase with the $\mathrm{H}$ component and the poloidal component in anti-phase (Sutcliffe and Lühr, 2010). Lastly, Pc1 waves with frequencies ranging between 0.2 and $5 \mathrm{~Hz}$ were observed in a pearls-on-a-string configuration. This Pc1 wave activity was highly localised and restricted to regions covering a relatively narrow $\mathrm{L}$ shells range (or differently, regions extending over $<100 \mathrm{~km}$, Engebretson et al., 2008). All these different types of ULF waves observed in the topside ionosphere are a complicated mixture of incident, reflected and mutually converted waves (Pilipenko et al., 2011).

For the quantitative monitoring of localised variations of power within time series of magnetic field data, wavelet spectral analysis has become popular (for examples see Balasis and Mandea, 2007; Balasis et al., 2012; Kunagu et al., 2013). Since the 1990s, the wavelet transforms is being more and more often applied as they offer a unique advantage over conventional Fourier transforms; thanks to their unique time localisation property, information is provided not only about the frequency of features present but also their location in the time series. Specifically, Balasis and Mandea (2007) used a technique employing wavelet transforms to look at CHAMP satellite data for ULF wave activity, while Heilig et al. (2007) developed an algorithm for the selection of possible ULF wave-related pulsation events (mainly Pc3) from both ground and space magnetometer data.

Previously, we had studied signatures of ULF waves in magnetic field collected by the CHAMP satellite in the topside ionosphere and these were compared with observations from two magnetospheric missions, Cluster and Geotail during the 2003 Halloween geospace magnetic storm (Balasis et al., 2012). Specifically, Pc3 along with Pc4-5 waves were reliably but manually identified by examining series of time-frequency spectrograms, produced with wavelet-based algorithms. The concurrent observations of Pc3 and Pc5 wave observations from the topside ionosphere to the outer magnetosphere revealed the potential of our wavelet-based algorithms for the analysis of multi-satellite magnetic field data and more importantly, the identification and classification of ULF waves.

In this paper, we describe a recently developed suite of wavelet-based algorithms suitable for the analysis of multiinstrument multi-satellite and ground-based measurements with the aim to detect ULF wave events in tri-axial satellite magnetic field data and distinguish them from processing errors or instrumental noise in an automated way. The timefrequency spectrogram forms the basis for the automated ULF wave detection and is used along with statistics derived from the time series data which convey information about the nature of the signal (e.g., amplitude of the filtered time series, spectral peak as well as frequency and wavelet power it corresponds, first derivative of the wavelet power). The first step consists of isolating short segments of the time series data where the wavelet power exceeds a predefined threshold power level. These are classified as wave "events" and "non-events" depending on whether a series of criteria are successfully met.

The applicability of the new wavelet-based algorithms is demonstrated with a case study. Pc 3 waves have been detected in tri-axial magnetic field data collected by the magnetospheric Cluster mission, the CHAMP and ST5 satellites flying in the topside ionosphere and the CARISMA network on the ground on 10 April 2006 between 19:59 UT and 20:43 UT. At the time, a magnetic storm which occurred on 9 April 2006 when $D_{\text {st }}$ reached a minimum valued of $-82 \mathrm{nT}$ was in progress. Nonetheless, it can also be applied for the detection of ULF waves observed within different frequency regimes, waves recorded using ground-based instruments or instruments onboard satellites (independently of whether satellite data is rotated into a field-aligned coordinate system).

The paper is organized as follows. Section 2 describes the dataset analyzed and the processing steps followed prior to analysis, while the beginning of Section 3 argues on the suitability of wavelet tranforms for detection and identification of ULF waves. In the rest of Section 3, we present our methodology, which consists of analysing ULF wave observations on time-frequency representation of magnetic and electric field measurements in combination with statistics derived from the time series. Lastly, in Section 4, we apply our algorithms on data from the CHAMP, ST5 and Cluster missions as well as the CARISMA network in a case study to discover the complexities of analyzing multipoint and multi-satellite observations. Section 5 summarises our findings.

\section{Data Selection and Processing}

Swarm has been selected by the European Space Agency (ESA) and scheduled for launch in 2013 with the aim to provide the most accurate survey of the Earth's magnetic field and its variations with time. For this purpose, one pair of satellites will be flying side-by-side in near-polar, circular orbits with an initial altitude and inclination of $450 \mathrm{~km}$ and $87.4^{\circ}$, respectively. The third satellite will be placed with $88^{\circ}$ inclination at an altitude of $530 \mathrm{~km}$ (Friis-Christensen et al., 2006). To ensure the unprecedented accuracy of measurements of the geomagnetic field collected by the three satellites, an absolute scalar magnetometer (ASM) is combined with a compact spherical coil (CSC) vector magnetometer sensor and a stellar compass to determine the attitude of the latter. Measurements of the magnetic field magnitude will be made available at resolution of $1 \mathrm{~Hz}$, while the resolution of the magnetic field vector measurements will be up to $50 \mathrm{~Hz}$.

In this paper, we have selected magnetometer data from 
the German LEO CHAMP satellite from 10 April 2006. The satellite had been launched in July 2000 into an almost circular, near-polar orbit (period: 94 minutes) with an initial altitude of $454 \mathrm{~km}$ (Reigber et al., 2002). Among the mission's objectives was to study the geomagnetic field and therefore, the satellite was equipped with a high performance fluxgate magnetometer (FGM) to measure the three components of the ambient magnetic field in the instrument frame. The FGM was combined with a star camera pair to determine the attitude of the assembly with respect to the stellar frame and an Overhauser scalar magnetometer serving as magnetic reference. Magnetic field data from the FGM with $1 \mathrm{~s}$ time resolution, provided in the sensor reference frame were supplemented by ion density data with 15 $\mathrm{s}$ resolution collected by the planar Langmuir probe (PLP).

In addition to magnetic field data collected by CHAMP on the topside ionosphere, we used $1 \mathrm{~s}$ time resolution magnetic field data in solar magnetic (SM) coordinates obtained by the 3 Space Technology 5 (ST5) microsatellites in orbit on 10 April 2006. Launched in March 2006, the 90 days mission of ST5 was primarily to test miniaturised satellites in the harsh environment of space and evaluate their ability to conduct scientific measurements. ST5 deployed into a $300 \times 4500 \mathrm{~km}$, dawn-dusk, and sun-synchronous polar orbit (period: 136 minutes) with $105.6^{\circ}$ inclination angle (Slavin et al., 2008). The combination of CHAMP and ST5 observations provided us with an approximation of measurements anticipated to be collected by the Swarm satellites flying in the topside ionosphere.

Heilig et al. (2007) had developed an algorithm for the selection of possible ULF wave-related pulsation events (mainly Pc3) from both ground and space magnetometer data, separately. To avoid a false interpretation of spatial structures as pulsation signals, resulting from the fast moving CHAMP satellite through the ambient field, they subtracted from the measurements the Potsdam Magnetic Model of the Earth (POMME) 2.5. This model includes the main field, the field of the ring current, large-scale magnetospheric fields, and the crustal anomalies up to spherical harmonic degree/order 90 (Maus et al., 2006). In particular, the removal of the last term was previously shown to be important for pulsation studies (Sutcliffe and Lühr, 2003; Vellante et al., 2004). However, one has to be aware that there are other magnetic signatures associated, for example, with plasma density fluctuations called equatorial spread-F (Stolle et al., 2006) or F-region current systems, which are not taken into account. Nonetheless, these signatures can disturb the estimation of pulsation activity.

We have opted to perform wavelet analysis of CHAMP FGM measurements without a priori processing of the data using field models, since the wavelet transform should in principle be capable of separating the various field source contributions. Similarly to Balasis and Mandea (2007), a high-pass filter was applied to data (thus removing the core field) prior to wavelet analysis. The purpose of filtering the time series was not only to emphasise short period signals, but also to distinguish between artificial noise and natural source signals.

ULF wave observations on the topside ionosphere were compared with those collected by the Cluster spacecraft in the inner magnetosphere. Cluster, which consists of 4 identical spacecraft that fly in tetrahedral configuration, was launched in 2000 with the aim to investigate the Earth's magnetic environment and its interaction with the solar wind (Escoubet et al., 1997). For this purpose, the Cluster spacecraft were originally placed in a $4 \times 19.6 \mathrm{R}_{E}$ elliptical polar orbit (period: 57 hours), with the single spacecraft orbits designed to enable a perfect tetrahedron to be formed at particular parts of the orbit. Cluster is the first space mission to feature multiple magnetometer datasets. For our study, we used magnetic field measurements from the FGM instrument (Balogh et al., 1997) with a time resolution corresponding to one spacecraft spin period, namely $4 \mathrm{~s}$ which have been provided in geocentric solar ecliptic (GSE) coordinates. Additionally, we used 4 s electric field measurements in the GSE coordinate system from the Electric Fields and Waves (EFW) instrument (Gustafsson et al., 1997).

A different approach was followed for calibrated data of the magnetic field from the four Cluster satellites. We have projected the $4 \mathrm{~s}$ FGM magnetic field measurements in a mean field-aligned (MFA) coordinate system which allows to separate ULF field variations perpendicular to as well as along the magnetic field direction. First, the average background magnetic field direction was estimated from a 20-min running average of the instantaneous magnetic field and along this, the parallel component. Next, the azimuthal component which is positive eastward and the meridional component, pointing radially outward at the magnetic equator were derived. Parallel, azimuthal and meridional components are referred to as compressional, toroidal and poloidal components, respectively. On the other hand, similarly to the magnetic field data from the CHAMP and ST5 satellites, a high-pass Butterworth filter with a cut-off frequency of $16 \mathrm{mHz}$ was then applied to finally obtain the wavelet power spectra covering the Pc3 frequency range (typically $20-100 \mathrm{mHz}$ ).

Ground magnetometers of the Canadian Array for Realtime Investigations of Magnetic Activity (CARISMA) chain are deployed across the North American continent (Mann et al., 2008). Fluxgate along with induction magnetometers record the three-dimensional (3D) vector magnetic field and its fluctuations at the surface of the Earth. In selecting data from the CARISMA network, we focused on the instance when CHAMP traversed over northern America and selected the magnetic station located closest to the footprint of its orbit on the ground. The magnetometer data from the Island Lake (ISLL) station at corrected geomagnetic latitude of $63.62^{\circ}$ and corrected geomagnetic longitude of $333.36^{\circ}$ are made available at $1 \mathrm{~s}$ sampling resolution in geomagnetic coordinates.

\section{Wavelet Transforms in the Study of ULF Waves}

Time-frequency analysis transforms a time series into a two-dimensional representation of frequency content with respect to time, allowing to study evolving signals. The Fourier transform identifies the frequency content of a signal by decomposing it as the sum of weighted sinusoidal functions of different frequencies. By windowing the time series and taking the Fourier transform of only a segment, 
we can identify changes in the frequency content from one segment to the next. The window length and overlap can be adjusted depending on the signal, but instantaneous estimation of spectral power cannot be provided. The wavelet transformation, instead of decomposing the signal into a basis of sine waves, decomposes the signal using temporally confined base functions, called wavelets, which allows for a much finer fit to the signal. The advantage of the wavelets over conventional sinusoidal functions is thus their time localization ability, providing information not only about the frequency of waves but also about their location in the time series.

A number of different methods have also been used in ULF wave analysis. Recently, the Hilbert-Huang transform (HHT), a nonlinear time-frequency analysis technique has been introduced to decompose the non-linear waveform of high-latitude geomagnetic pulsations into different wave modes such as Pi1, Pi2 and Pc3 waves (Kataoka et al., 2009). Maximum Entropy Spectral Analysis (MESA) is a different nonlinear method which has been used by Ndiitwani and Sutcliffe (2009) to compute the dynamic spectra of Pc3 waves observed by the CHAMP satellite as well as on the ground. On the other hand, the WignerVille distribution, and related refinements, offers a class of time-frequency analysis tools that are distinguished from Fourier and wavelet transforms by superior temporal and frequency resolution in the time-frequency plane (Chi and Russell, 2008). Complex demodulation has been occasionally used for its ability to provide instantaneous estimates of the waves amplitude, phase and polarization at a specific frequency through comparison with a reference signal (i.e., Lee et al., 2007).

Despite the multitude of time-frequency analysis methods which have been proposed over the years, a series of arguments supports the selection of the wavelet analysis for ULF wave studies. The frequencies of Pc3 wave events to be investigated are some tens of $\mathrm{mHz}$ and therefore, magnetic field data with a few $\mathrm{mHz}$ resolution are needed. Using the Fourier transform, this would require 15-20 minutes intervals of data. However, since a LEO satellite such as CHAMP was moving with a speed of $4^{\circ}$ lat./min, these long intervals corresponding to latitudes ranging from $60^{\circ}$ to $80^{\circ}$ would have made it impossible to investigate the spatial structure of these waves (Heilig et al., 2007). Nonetheless, the wavelet analysis proved suitable to overcome this problem, as it has the ability to measure all the frequencies on their appropriate scales.

Specifically, a previously performed wavelet analysis covering approximately three years (August 2000-May 2003) of $1 \mathrm{~Hz}$ magnetic field data collected by the FGM instrument onboard the CHAMP satellite gave promising results (Balasis et al., 2005). The wavelet based analysis of CHAMP data was able to detect, identify and classify artificial noise sources, such as instrumental problems and pre-processing errors as well as high frequency natural signals of external fields, such as F-region ionospheric plasma bubbles (see Stolle et al. (2006) for a statistical analysis of these events) and lastly but more importantly, magnetospheric Pc3 waves.

\subsection{The time-frequency analysis (TFA) tool}

In this paper, we present an automated time-frequency analysis (TFA) tool based on the continuous wavelet transform and the Morlet function is used as the wavelet basis function (Fig. 1). On the selection of an appropriate mother function, we have tested some of the most commonly used in the literature and concluded that the best options are the "Paul" and "Morlet" functions. Specifically, the Paul function has a better accuracy in the temporal dimension, while the Morlet function is more accurate in the frequency domain. Since we are more interested in the accurate frequency decomposition of waves, we have opted in favor of the latter, though the results were checked with both for consistency.

We should also note that there are several parameters of the wavelet transform (e.g., frequency range, power spectral density amplification factor, etc.) which need to be correctly adjusted in order to capture different characteristics of the signal. For example, a low value of the non-dimensional frequency parameter of the Morlet wavelet, $\omega_{0}=5-10$, gives high time resolution, appropriate for the study of the Pc4-5 waves. However, a higher value, $\omega_{0}=16-20$, is better suited for Pc3 waves, when it necessary to more accurately determine the pulsations frequency, as we do in this paper. On the other hand, the power scaling factor, a multiplier to be applied to the estimated wavelet power spectra, for normalization purposes or to enhance the spectra on cases of very weak activity, is set with test data sets.

The input and output of the new wavelet analysis tool on satellite magnetic field measurements are described below:

1) daily data files of magnetic field measurements with different time resolutions are received as input; and

2) wavelet power spectra of the total magnetic field for each half-orbit (i.e. as the satellite flies from a geomagnetic latitude of $-90^{\circ}$ to $+90^{\circ}$ ) are produced as output.

In detail, for the case of the CHAMP mission, which takes approximately 45 minutes to complete a half-orbit, this MATLAB routine produces 32 figures per day. Typical examples are being given in Figs. 2-4, in which no latitudinal constraint on the analysis of the satellite data was imposed. The figures produced consist of three graphs:

1) the upper panel showing the filtered time series of the magnetic field magnitude;

2) the second panel with the corresponding power spectrum produced from wavelet analysis;

3) a third panel with the time variation of the PLP measurements, providing information about the particle population along the satellite's orbit (y axis on the left and black line on the graph) together with the magnetic latitude of the satellite as a function of time, coupled with the magnetic local time (MLT);

all about the corresponding time period in which the satellite is in the user-defined area of interest, and continue giving such plots for every passing until the input data are exhausted (usually data spans a 24 hours time period). In the wavelet power spectrum of Fig. 4, ULF wave activity is clearly seen starting approximately at 20:08 UT and last- 


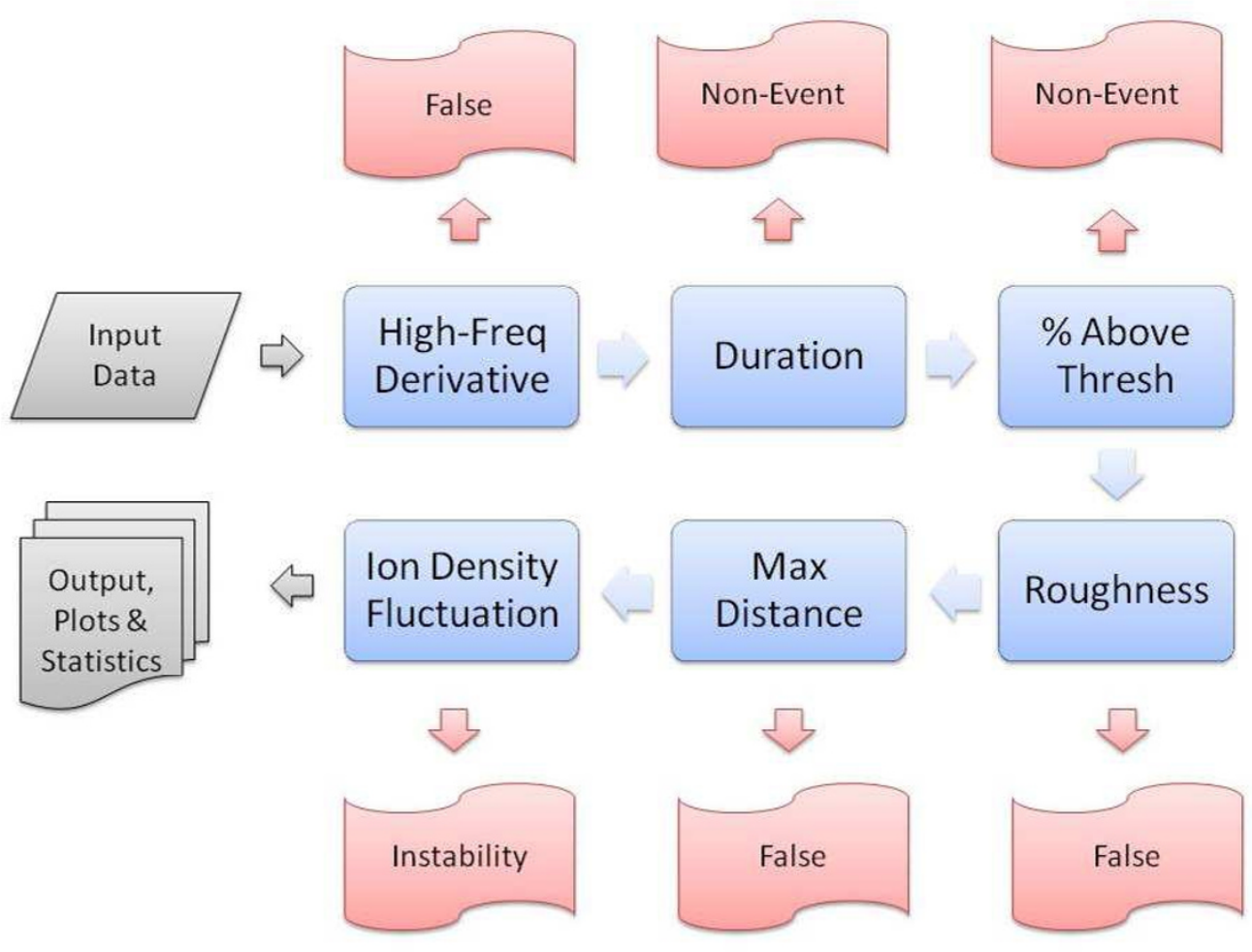

Fig. 1. Flowchart of the Detection Process. Each candidate is tested against a series of criteria (blue boxes) and if it passes, it is being forwarded to the next. If not, it is assigned to the appropriate category (red, outer boxes) based on which test it failed to pass. To be characterized as an actual Event, a candidate must meet all criteria, in which case it is passed to the output.
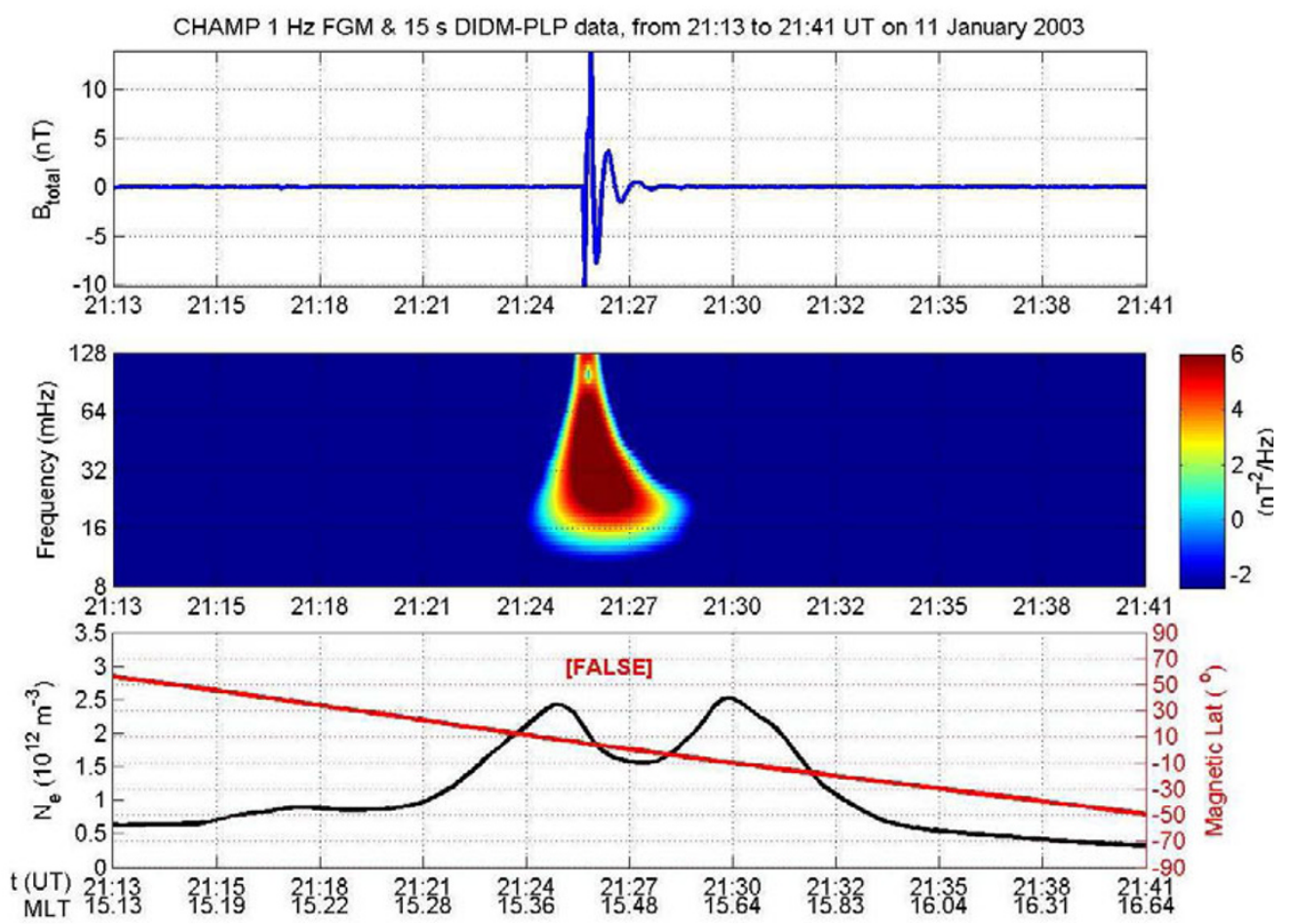

Fig. 2. CHAMP track with a step discontinuity. From top to bottom, CHAMP filtered magnetic field time series, its corresponding wavelet power spectrum and the combined PLP data / magnetic latitude plot, all with respect to time (UT) for a satellite pass between 21:13 and 21:41 UT on 11 January 2003. The bottom row provides additional information about the satellite's MLT position. The noise is marked with a '[FALSE]' (in red) flag in the lower panel. 

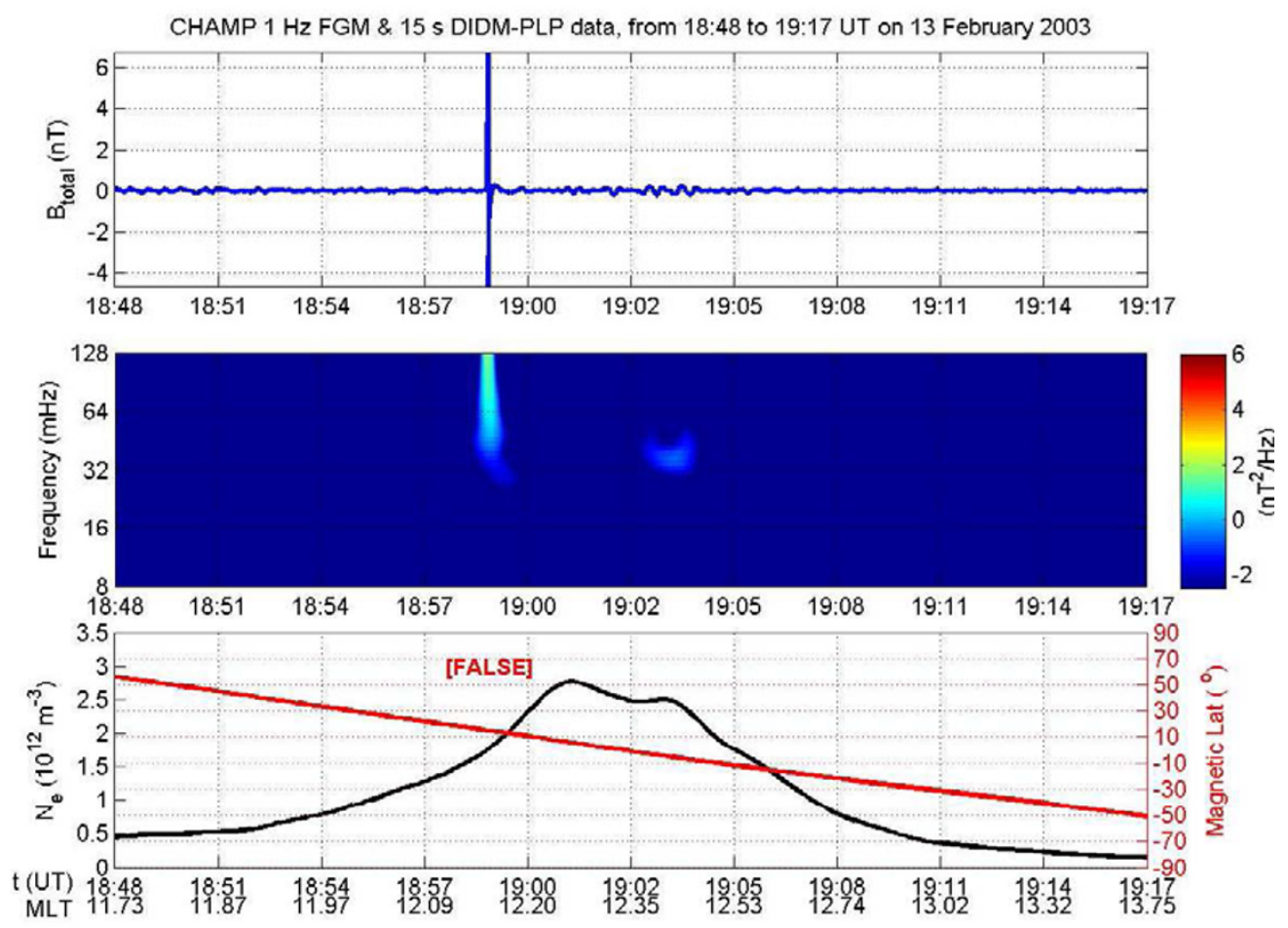

Fig. 3. CHAMP track with a spike. As in Fig. 2 but from 18:48 to 19:17 UT on 13 February 2003. The noise is also marked here with a '[FALSE]' (in red) flag in the lower panel.
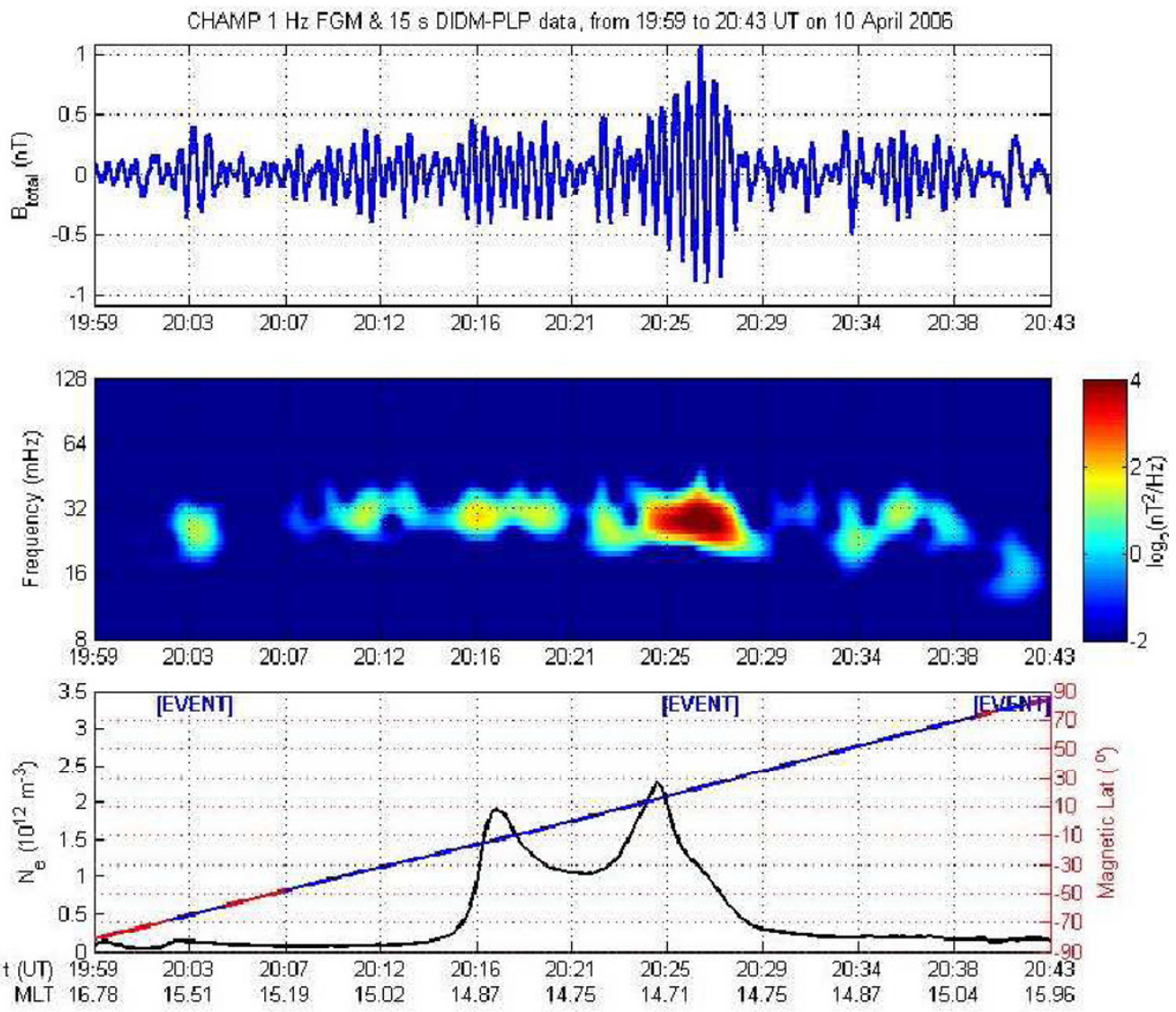

Fig. 4. CHAMP track with a Pc3 wave event. As in Fig. 2 but from 19:59 to 20:43 UT on 10 April 2006. The wave activity is marked with '[EVENT]' (in blue) flags in the lower panel. The latitudes where the ULF activity is observed are also given in blue in the same panel. 
ing for almost half an hour on 10 April 2006, namely in the recovery phase of the storm occurred on 9 April 2006 when the $D_{\text {st }}$ was $\sim-30 \mathrm{nT}$. The peak power is detected between 22 to $32 \mathrm{mHz}$, in the frequency range of Pc3 waves.

The wavelet power range was selected in this example to facilitate comparison between different natural or artificial signals and the observations from the ground and space magnetometer data presented in the next sections. It was set to improve the visualization of results. Within the TFA tool, a different power range can be used for each space mission and ground observatory and needs to set empirically to cover most cases of activity. It is true, that for some rare and extreme cases, there will be saturation, but those cases can then be examined separately with more detail.

While there is an option to remove the local magnetic field (for LEO satellites such as CHAMP) with the use of a model of the Earth's magnetic field or differently estimate the cubic fit on the time series segment to be analyzed, in this example, we have chosen to perform wavelet analysis of CHAMP FGM measurements without a priori processing using magnetic field models. As it has been detailed in Section 2, with the application of a high pass filter, the slower variations will be removed and short period signals, namely Pc3 waves will be revealed.

\subsection{Automated detection of ULF wave events}

In this section, we use three time series of the total magnetic field and ion density data from the CHAMP satellite each covering 24 hours. Specifically, FGM and PLP data from 11 January 2003, 13 February 2003 and 10 April 2006 are used to demonstrate the procedure followed to detect and characterize ULF wave events. The data are sampled at a frequency of $1 \mathrm{~Hz}$, namely the sampling frequency they are provided and are processed in blocks of 1 day. Our methodology consists of the following steps (Fig. 1).

We use the wavelet transform implemented in a conventional dynamic spectrogram as the starting point. First, we scan the wavelet power spectrum along the temporal dimension to find consecutive points for which the wavelet power exceeds a predefined threshold. We mark these intervals as "Candidate events" and extract a number of crucial signal parameters from the wavelet power spectrum and the original time series. Specifically, the parameters derived from the wavelet power spectrum include: onset and duration of the "Candidate events" as well as number of points for which the wavelet power is above the predefined threshold, value of total wavelet power but also of average wavelet power over the isolated intervals, spectral peak as well as frequency and wavelet power it corresponds, occurrence time of peak frequency and first derivative of wavelet power at the highest frequency, and frequency range where wavelet power is enhanced.

In other words, our algorithm has been designed having in mind the way a human would manually detect wave activity by looking at a wavelet spectra; namely, by looking for portions of the wavelet spectrum where wavelet power appears to be significantly higher than in the surrounding and the frequency around which the enhancement in wavelet power is centred varies with time in some regular way. Nonetheless, both step changes and spikes present in the magnetic field time series are transformed during fil- tering, which results in a characteristic pattern of a damped sinusoidal oscillation of varying frequency. A significant part of the algorithm is devoted to detecting exactly these features in the wavelet power spectrum and classify them as a "False Positive" signal.

For this purpose, from the filtered time series the maximum amplitude of signal is extracted along with the difference of maximum amplitude from the mean value, measured in standard deviations of the time series, and lastly the signal roughness (average absolute value of its second derivative at local extrema). To these parameters, the roughness of the ion density time series is added as well as information about the magnetic latitude of the satellite and magnetic local time the observations correspond. We use the classification schema which is illustrated in Fig. 1 and is based on this set of parameters to examine if the "Candidate events" passes successfully six criteria to be marked as an actual "Event". In case of failure, we identify the candidate track as a "False Positive" or "Non-Event". We plot the track with the appropriate flags, classify it and save relevant statistics.

We examine the efficacy of our methodology in the presence of a step change and a spike in the magnetic field time series in Figs. 2 and 3, respectively. Short lived bursts and spikes in the magnetic field data tend to result in enhancements of the wavelet power but they exhibit none of the characteristics typical to wave phenomena. A major difference lies in the fact that these "False events" are characterized by enhanced wavelet power at so high frequencies as $250 \mathrm{mHz}$. Futhermore, the wavelet power increases and decreases rapidly at the high frequencies. As a consequence, their detection and proper classification as "False events" is possible by examining the time derivative of their wavelet power and checking whether it exceeds a predefined threshold. Similar behavior is expected in cases of discontinuities in the time series, such as data gaps, which are being translated through filtering in artificial oscillations. Even these oscillations in the filtered time series exhibit a sudden increase of the wavelet power at high frequencies and therefore, can be classified according to the same criterion (criterion \#1).

Unusually short duration of wavelike events is also a valid indication that "Candidate events" do not correspond to actual wave activity and so is a low number of points with wavelet power above the predefined threshold, suggesting extremely weak activity associated with background noise (criteria \#2 and 3). The next criterion (criterion \#4) employed is based on the roughness of the magnetic field time series to capture filter ringing effects which are characterized by the artificial oscillation in the filtered time series which appears "too smooth" to correspond to a natural signal. "Measures of roughness" employed are the average second time derivative of the time series, computed only in cases of local extrema, and the time series fractal dimension computed by means of the Box Counting method.

Lastly, "Candidate events" corresponding to areas of intense fluctuations in the ion density may be attributed to the presence of equatorial spreads in the $\mathrm{F}$ region of the ionosphere, or in general plasma instabilities. To identify these cases and classify such special events as "Instabilities", the 


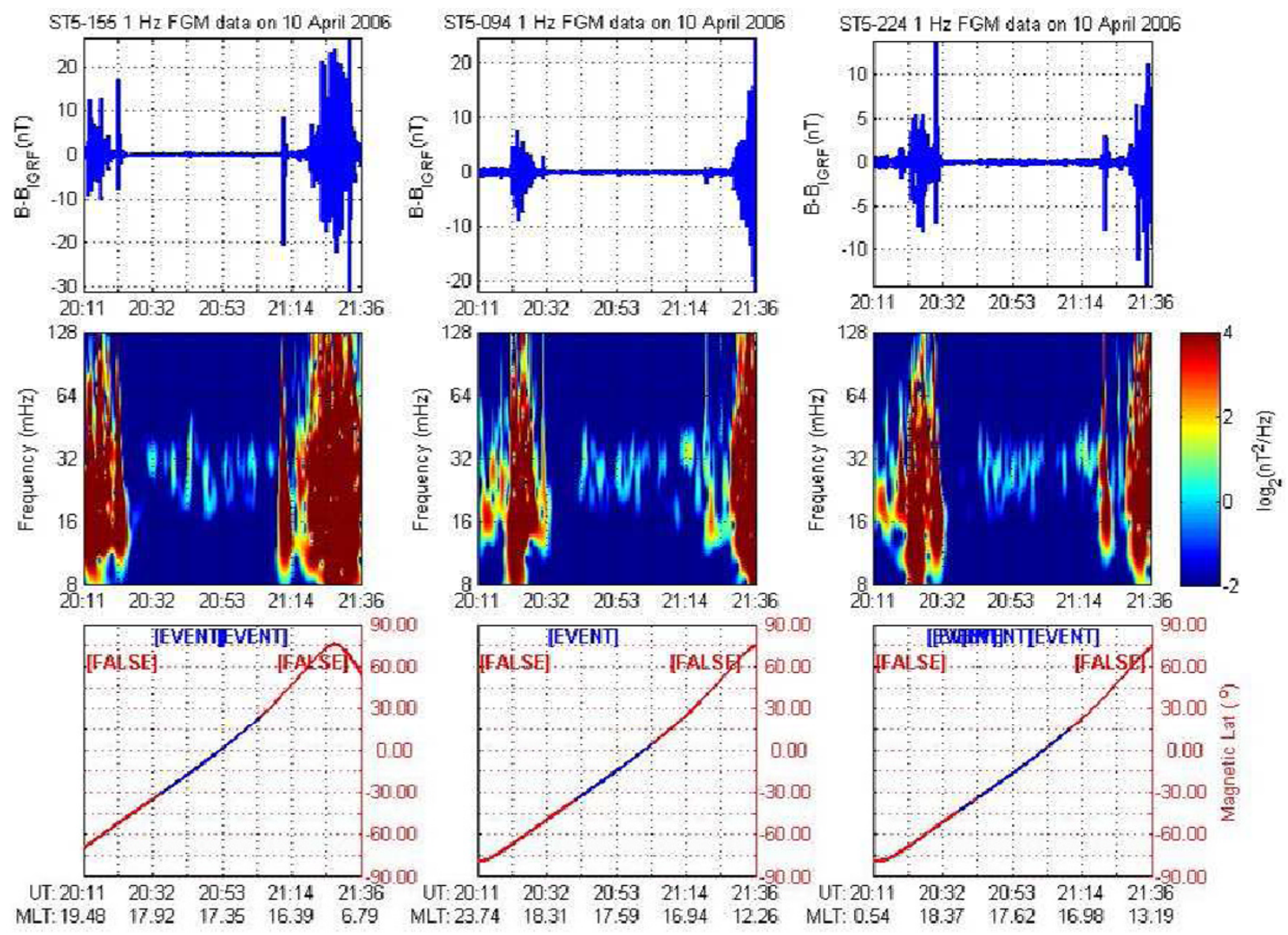

Fig. 5. A three-column figure, presenting the output of the time-frequency analysis tool for the three-satellite case, using data from ST5 155,094 and 224 satellites (after subtracting IGRF model) at the closest pass in time with the CHAMP track of Fig. 4. From top to bottom, filtered magnetic field, wavelet power spectrum, and magnetic latitude vs time. The bottom row at each column provide additional information about the satellites' MLT position. Flagging of noisy segments and wave events in lower panels as in Figs. 2-4.

roughness of the ion density time series is used (criterion \# 6). Only if all six criteria are successfully met, the segment of the wavelet power spectrum isolated as "Candidate event" is flagged as an "Event". An example of such a successfully detected event is given in Fig. 4, which is analyzed in detail in Section 4.

Obviously, the way in which the algorithm separates ULF wave events is somehow arbitrary and different values of the initial wavelet power threshold may yield different results. The decision in favor of a rather lenient first threshold is based on the fact that in this manner even short and weak magnetic field oscillations can be detected, while a stricter threshold would risk splitting ULF wave events of rather long duration to multiple shorter ones. It is worth noting also that all parameters are already computed before ULF wave events are classified as such. Thus, useful statistics can be derived for each and every category of events, giving valuable insights on plasma instabilities as well as indications on the general wave activity with respect to time.

\section{Demonstration of the TFA Tool}

Figure 4 presents the CHAMP total magnetic field filtered time series and Pc3 wave dynamic spectrum for the time interval from 19:59 to 20:43 UT on 10 April 2006. The third panel in Fig. 4 shows the electron density data derived from the $15 \mathrm{~s}$ PLP measurements. The corresponding values of the CHAMP magnetic latitude and MLT are also provided in that panel, showing that the satellite was located in the dayside. The inclusion of the electron density recordings helps to identify the time segments of the signal that contain signatures of the post-sunset equatorial spread F (ESF) events (Stolle et al., 2006) and discriminate between Pc3 wave and plasma depletion occurrence. In the wavelet power spectrum, ULF wave activity is clearly seen starting approximately at 20:08 UT and lasting for almost half an hour, namely in the recovery phase of the storm occurred on 9 October 2006 when the $D_{\text {st }}$ was $\sim-30 \mathrm{nT}$. The peak power is detected between approximately 22 to $32 \mathrm{mHz}$, in the frequency range of Pc3 waves.

In Fig. 5, we provide an example of the applicability of the tool on measurements from a LEO multi-satellite mission using data from the three satellites of the ST5 constellation (SC-155, SC-094 and SC-224) collected during 10 April 2006. The plots drawn from top to bottom are the filtered total magnetic field, after subtracting the corresponding IGRF model (Macmillan and Maus, 2005), wavelet power spectrum, and magnetic latitude versus time. The $\mathrm{X}$ axis of the plot at the bottom of each column provides information about the Universal Time (UT) as well as the respective satellites' MLT position. Due to constellation's 

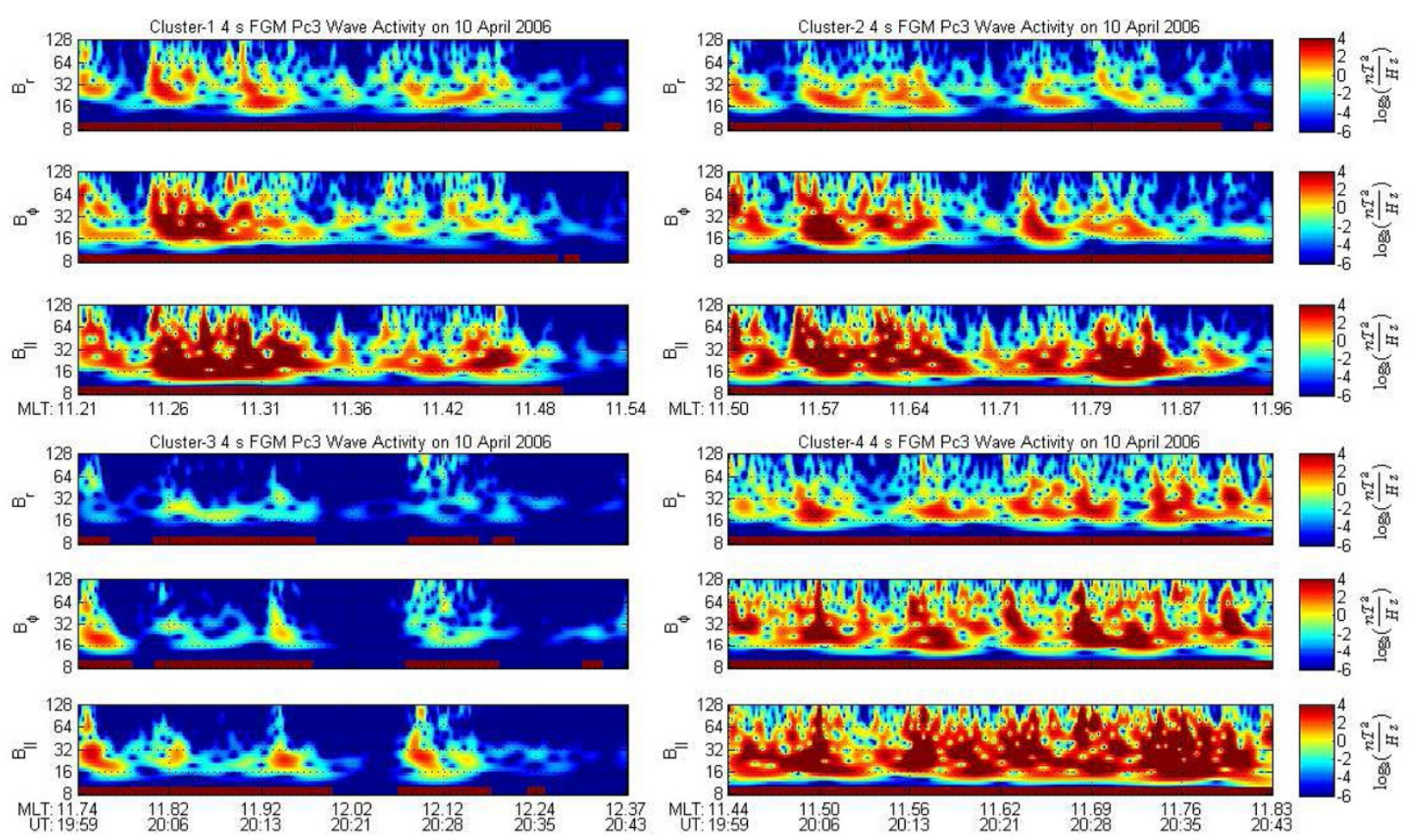

Fig. 6. This multiple figure is a combined plot, presenting the output of the TFA tool for the magnetic field in the Pc3 band, as measured by all Cluster spacecraft at the same time interval as with CHAMP track of Fig. 4. Here, the wavelet power spectra of the poloidal, toroidal and compressional components of the field are shown. Red bars at the bottom of each panel indicate time intervals with wave activity.
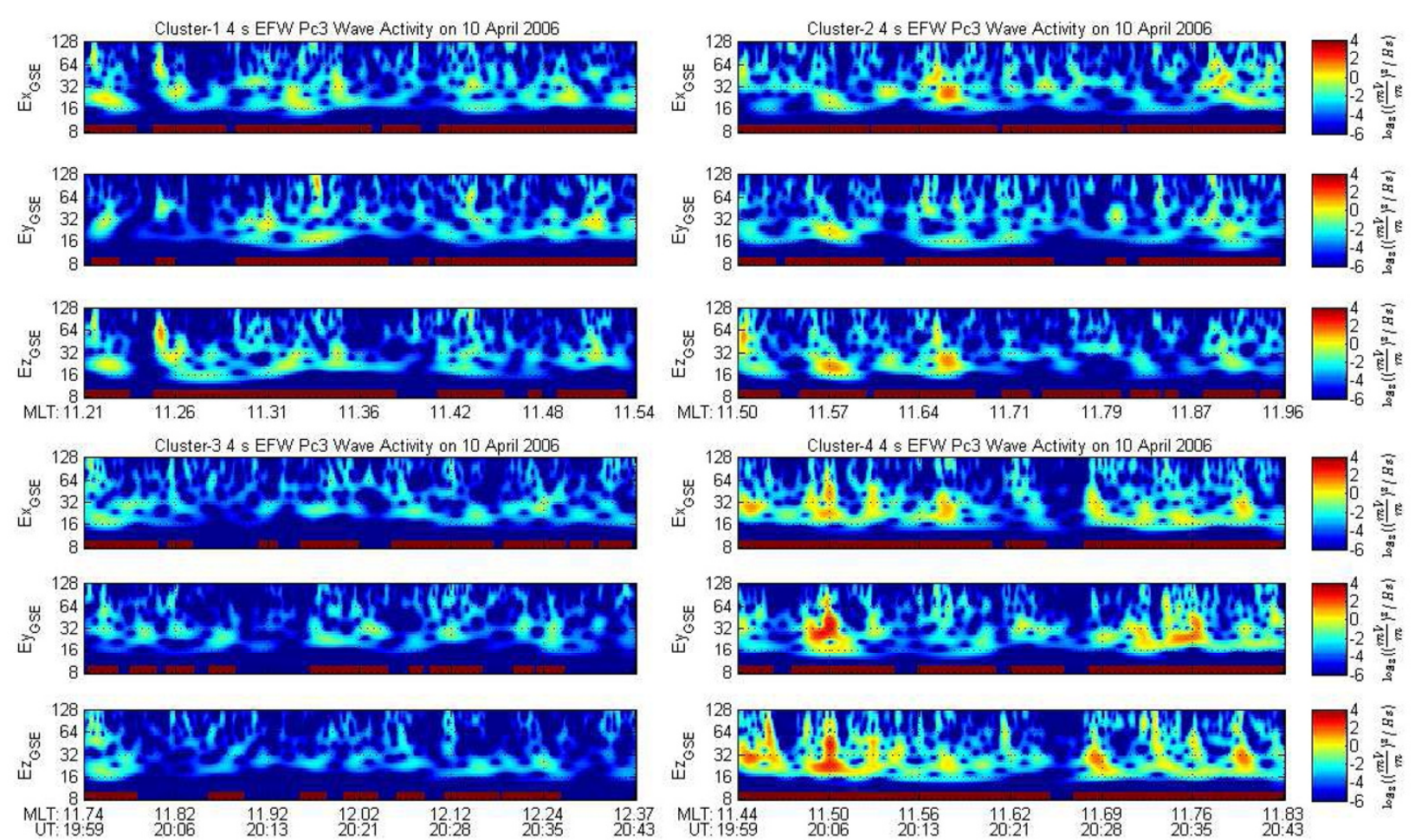

Fig. 7. This multiple figure is a combined plot, presenting the output of the TFA tool for the electric field in the Pc 3 band, as measured by all Cluster spacecraft at the same time interval as with CHAMP track of Fig. 4. Here, the wavelet power spectra of the X, Y and Z GSE components of the field are shown. Red bars at the bottom of each panel indicate time intervals with wave activity. 


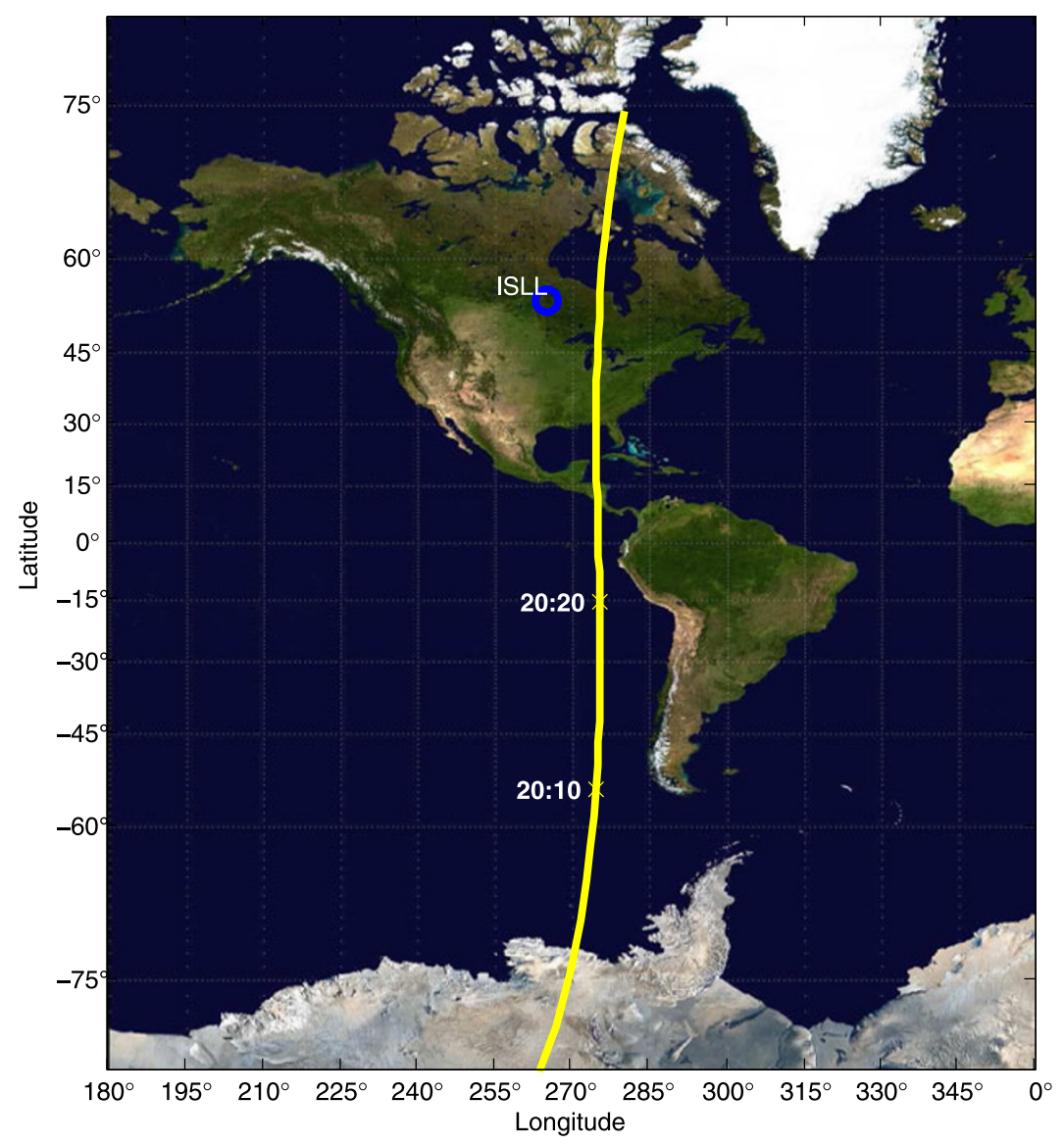

Fig. 8. Map showing the projection of CHAMP track onto the ground (in yellow) and the location of Island Lake (ISLL) station.

formation, ST5-155 track lasts from 20:00 to 21:28 UT, ST5-094 track starts at 20:11 and ends at 21:36 UT and ST5-224 track lasts between 20:13 and 21:38 UT. All plots indicate prominent Pc3 activity $(22-32 \mathrm{mHz})$ at approximately 20:30 to 21:00 UT. Between 20:00 and 21:00 UT, the ST5 constellation was near its apogee at the dawnside, with the leading satellite located at $67^{\circ}$ latitude and $112^{\circ}$ longitude, while the trailing satellites were flying at approximately $73.5^{\circ}$ latitude and $157^{\circ}$ longitude, passing from the nightside to the dayside magnetosphere and from the southern to the northern hemisphere.

Figures 6 and 7 show composites of dynamic wavelet power spectra from 19:59 to 20:43 UT (for the same time interval as with CHAMP track) in the Pc3 frequency range for all Cluster spacecraft. In Fig. 6 we provide the results from the application of TFA tool on the magnetic field measurements of Cluster-1, 2, 3 and 4 after the latter were transformed into an MFA coordinate system (i.e., poloidal, toroidal and compressional components). In Fig. 7 we provide the results from the application of TFA tool on the electric field measurements of Cluster-1, 2, 3 and 4 in the Geocentric Solar Ecliptic (GSE) coordinate system (i.e., X, $\mathrm{Y}$ and $\mathrm{Z}$ components). It should be noted that the Cluster constellation had crossed the magnetopause and was heading towards the perigee during the time interval between 20:00 and 21:00 UT on 10 April 2006. At approximately 20:30 UT, the four satellites were located at a distance of 12 to $13.5 R_{E}$, with the 3 first around $(1,-6.5,-10.5) R_{E}$, in XYZ GSE coordinates and the Cluster-4 lagging behind at about $(1.27,-7,-11) R_{E}$. Figure 6 shows that there is intense activity seen for the whole time interval and at all frequencies by the Cluster-4 satellite, while Cluster- 3 seems to be less contaminated by signals (both in a temporal and spectral manner) in comparison to the rest of mission's spacecraft. As expected the dynamic wave power spectra of the electric field data for the four spacecraft (Fig. 7) are much more noisy than the corresponding spectra of the magnetic field (Fig. 6).

In order to examine observations from ground stations corresponding to satellites' location, we examine the CHAMP footprints between 19:59 and 20:43 UT on 10 April 2006. The CHAMP satellite was flying in the dayside topside ionosphere, from the southern to the northern hemisphere and at 20:30 UT was located at $37.6^{\circ}$ geographical latitude and $275.2^{\circ}$ geographical longitude, with its footprint lying at $38.5^{\circ}$ latitude and $275^{\circ}$ longitude (Fig. 8). The search for the footprint of CHAMP on the Earth's surface led us to the Island Lake station (found at $53.86^{\circ}$ latitude, $265.34^{\circ}$ longitude) located along the Churchill Line of the CARISMA network (Fig. 8). After applying a 16 $\mathrm{mHz}$ high-pass filter to the $1 \mathrm{~Hz}$ FGM measurements $(\mathrm{H}$, $\mathrm{D}$ and $\mathrm{Z}$ components) of this station, we have derived their corresponding wavelet power spectra in the Pc3 frequency band.

Figure 9 presents a composite of dynamic wavelet power spectra, covering the Pc3 wave activity as seen by the 


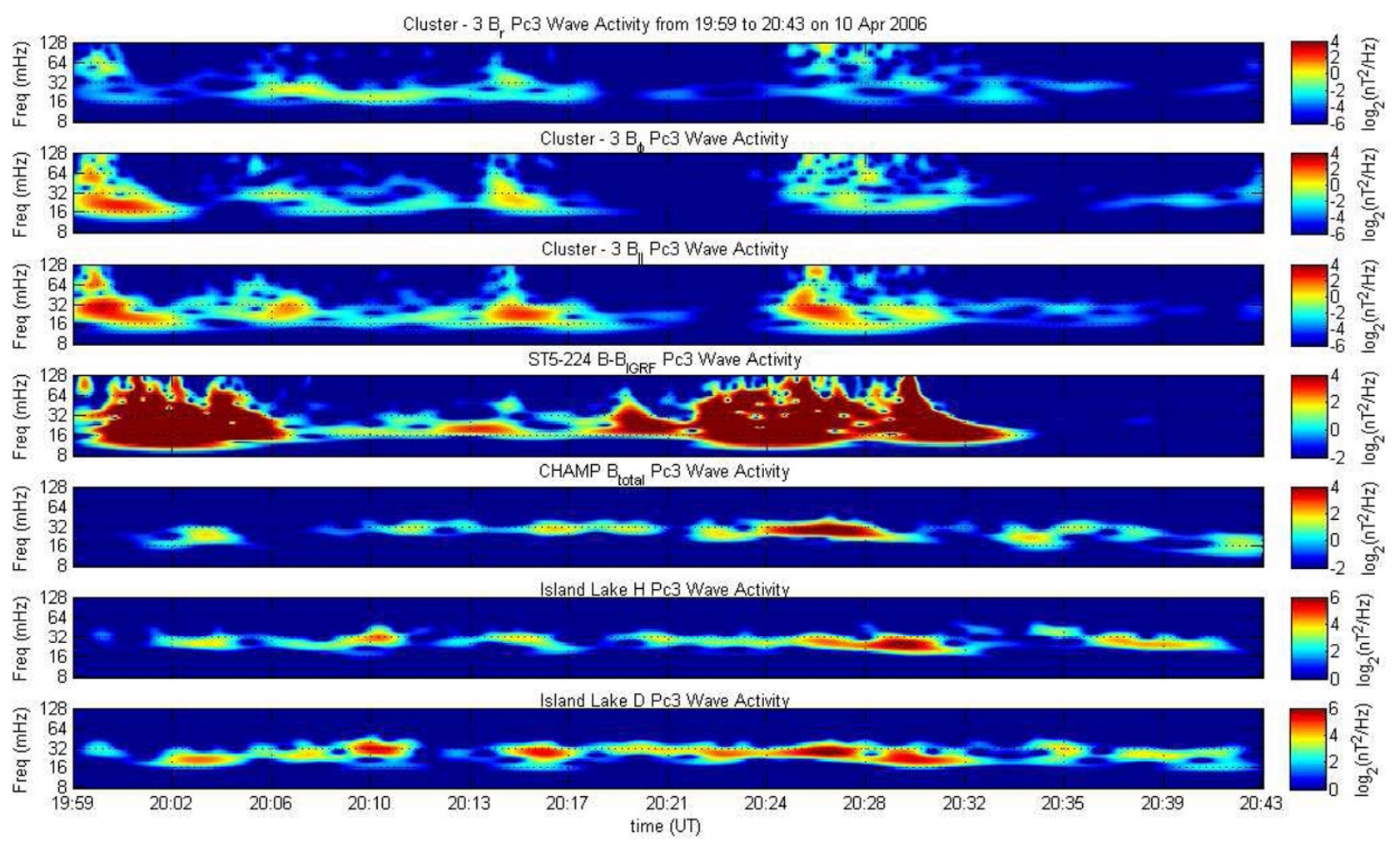

Fig. 9. From top to bottom, the wavelet power spectra of the Pc3 wave activity from Cluster-3 MFA components, ST5-155 total field (after subtracting IGRF model), CHAMP total field, and Island Lake H and D components, between 19:59 and 20:43 UT on 10 April 2006.

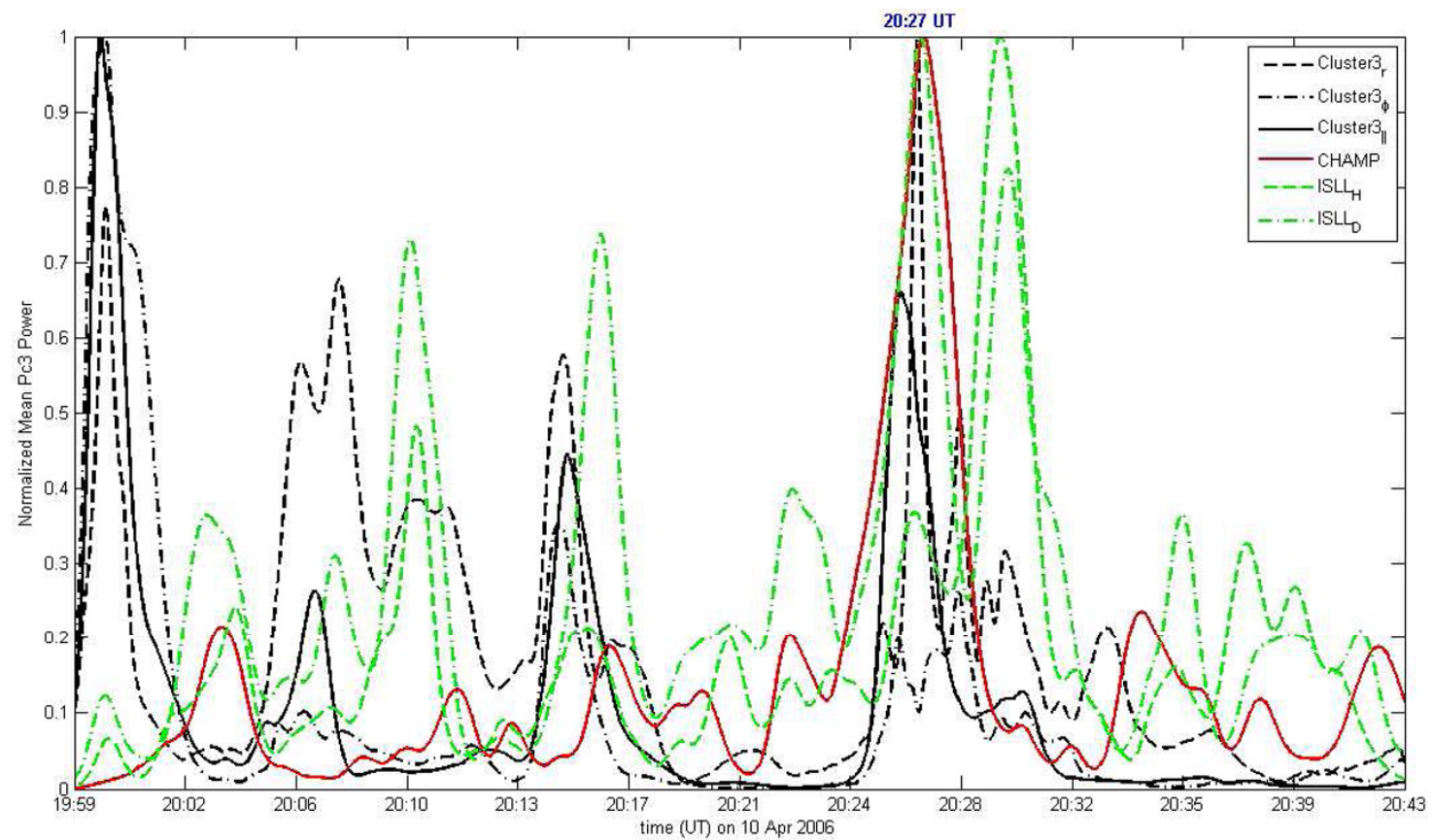

Fig. 10. The normalized average power in the Pc3 frequency range derived from the wavelet power spectra of Fig. 9 for Cluster-3 MFA components, CHAMP total field, and Island Lake H and D components, between 19:59 and 20:43 UT on 10 April 2006. We clearly observe coincident maximum values of wave power at 20:27 UT for Cluster-3 poloidal, CHAMP total and Island Lake D component.

Cluster-3, ST5-155 and CHAMP satellites as well as at the Island Lake ground station between 19:59 and 20:43 UT on 10 April 2006. In particular, Fig. 9 shows the wavelet power spectra of the poloidal, toroidal and compressional components for Cluster- 3 in the dayside magnetosphere and the total field for ST5-155 and CHAMP located at $\sim 18$ MLT and 15 MLT, respectively. Additionally, we depict the $\mathrm{H}$ and D components for the CARISMA station. In Fig. 10 we show the normalized average power in the Pc3 frequency range derived from the wavelet power spectra of Fig. 9 for 

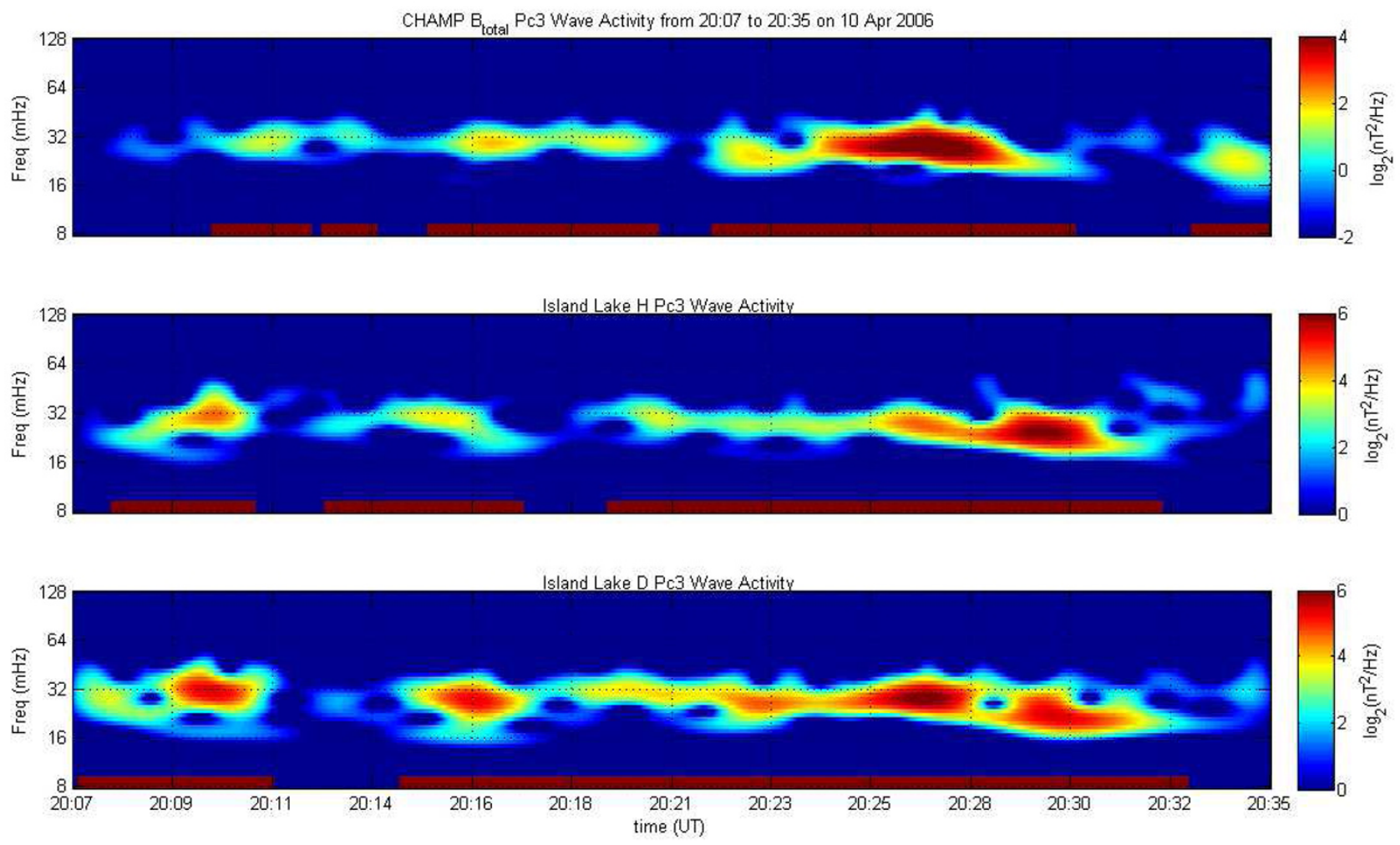

Fig. 11. This figure zooms in on Fig. 9. From top to bottom, the wavelet power spectra of the Pc3 wave activity from CHAMP total field, and Island Lake H and D components, between 20:07 and 20:35 UT on 10 April 2006. Red bars at the bottom of each panel indicate time intervals with wave activity.

Cluster-3 MFA components, CHAMP total field, and Island Lake H and D components, between 19:59 and 20:43 UT on 10 April 2006. Simultaneous peak values of wave power at 20:27 UT are evident for Cluster-3 poloidal, CHAMP total and Island Lake D component. This demonstrates the ability of the TFA tool to detect coherent ULF wave signatures at various platforms in different locations. Moreover, Fig. 11 focuses on the time interval from 20:07 to 20:35 UT on 10 April 2006, showing power spectra for the CHAMP satellite (total field) and Island Lake station (H and D components). Figure 11 shows that CHAMP and Island Lake simultaneously observe Pc3 wave activity at the frequency range between 22 and $32 \mathrm{mHz}$ with peak wave power seen approximately around 20:25 UT (the peak power is probably seen a couple of minutes earlier in CHAMP data and on the ground $\mathrm{D}$ component in comparison to $\mathrm{H}$ component).

\section{Discussion and Conclusions}

This paper describes a time-frequency analysis tool for automated detection of magnetospheric ULF wave events in magnetic and electric field observations made from multisatellite missions and ground-based networks.

This new tool has been based on the experience gained through the analysis of magnetic field data collected by the CHAMP mission in combination with data from the Cluster mission, the results of which have been presented in our previous paper (Balasis et al., 2012), where ULF waves were reliably but manually identified by examining series of time-frequency spectrograms, produced with wavelet-based algorithms. The technique developed for the detection of
ULF waves is simple, but we think that it could be useful for the analysis of observations expected to be collected by the Swarm mission concurrently with the Cluster mission. The case study has been added to demonstrate its capabilities as these have been tested so far.

Focusing on the 3-month interval that data from the multi-satellite LEO mission of ST5 are available (MarchJune 2006), we show an application of our tools relevant to the moderate magnetic storm of 9 April 2006 (with $D_{\text {st }}$ minimum $-82 \mathrm{nT}$ ). First, we find a clear signature of a Pc3 wave event in the magnetic field recordings of the topside ionosphere CHAMP satellite that occurred in the recovery phase of the storm. Then, we proceed to examine simultaneous magnetic field measurements from the multi-satellite topside ionosphere mission ST5. Additionally, we look to magnetic and electric field data from Cluster 4-spacecraft mission for the same event and check the ground magnetic station Island Lake of the CARISMA array, which is the closest station to the CHAMP's footprint. CHAMP and Island Lake analysis results show evidence for simultaneous observed Pc3 wave activity from 20:07 to 20:35 UT at the frequency range between 22 and $32 \mathrm{mHZ}$ with peak intensity centered approximately around 20:25 UT (the peak wave power is probably seen earlier in CHAMP data and on the ground D component in comparison to $\mathrm{H}$ component).

An interesting result arises by performing some additional analysis that involves calculations of the average Pc3 power from the original wavelet power spectra in the magnetosphere (Cluster), topside ionosphere (CHAMP) and Earth's surface (Island Lake). Coincident maximum val- 
ues of wave power are evident at 20:27 UT for Cluster-3 poloidal, CHAMP total field and Island Lake D component. These coherent Pc3 wave signatures indicate that our tool can facilitate monitoring of a ULF wave event in different locations of geospace. We note that this particular event deserves further attention, because multi-point wave observations are usually made when different satellites are in good local time conjunction, while Cluster and CHAMP were not in such a conjunction during this event.

Thanks to TFA tool's ability to distinguish magnetospheric pulsations from processing errors and / or instrumental noise, the scientific merit for the large (external and internal field) researchers' community interested in the Swarm mission would expected to be at various levels:

(a) Capability to identify artificial source noise in the time series.

(b) Capability to detect external source natural signals in the time series.

(c) Applicability both to multi-satellite and single satellite missions flying in different regions of the magnetosphere and topside ionosphere (i.e., opening opportunities for Cluster-Swarm combination or synergetic studies).

(d) Applicability both to spaceborne and multiple ground data.

(e) Applicability both to magnetic and electric field measurements as well as particle data.

The above mentioned characteristics are equally important for internal field modellers (e.g., data selection) as well as space physicists (e.g., ULF wave events and equatorial spread-F (ESF) events statistics).

Scientifically useful analysis of in situ ULF wave data also requires analysis of coherence (or coherency), phase difference, polarization parameters such as azimuth angle, ellipticity and degree of polarization. In addition the Poynting flux is extremely useful. At the very least, coherence is an important parameter, widely used in other multipoint in situ and ground-satellite studies (e.g., Takahashi et al., 1990; Kim and Takahashi, 1999; Teramoto et al., 2011). The derivation of polarization and propagation parameters in a field-aligned coordinate system is already part of our work in progress and there are plans to implement these features in the TFA tool. So far we have experimented with techniques based on either the eigenvalue or singular value decomposition of the field components' spectral matrices, as described in Samson (1973) and more recently by Santolik (2003). We already have encouraging initial results and are confident that these methods will be an integral part of next versions of our tool.

Acknowledgments. This work has received funding from the European Space Agency under contract ESTEC 4000103770/11/NL/JA/ef and European Union's Seventh Framework Programme (FP7-SPACE-2011-1) under grant agreement $n$. 284520 for the MAARBLE (Monitoring, Analyzing and Assessing Radiation Belt Loss and Energization) collaborative research project. We thank two anonymous referees for their constructive comments. We gratefully acknowledge ESA's Cluster Active Archive, NASA's Virtual Magnetospheric Observatory, Helmholtz Centre Potsdam GFZ, German Research Centre for Geosciences, and the Canadian Space Agency, for supplying data used in this study.

\section{References}

Balasis, G. and M. Mandea, Can electromagnetic disturbances related to the recent great earthquakes be detected by satellite magnetometers?, special issue "Mechanical and Electromagnetic Phenomena Accompanying Preseismic Deformation: from Laboratory to Geophysical Scale", edited by K. Eftaxias, T. Chelidze and V. Sgrigna, Tectonophysics, 431, doi:10.1016/ j.tecto.2006.05.038, 2007.

Balasis, G., S. Maus, H. Lühr, and M. Rother, Wavelet analysis of CHAMP flux gate magnetometer data, in Earth Observation with CHAMP, edited by C. Reigber, H. Lühr, P. Schwintzer and J. Wickert, 347-352, Springer, New York, 2005.

Balasis, G., I. A. Daglis, E. Zesta, C. Papadimitriou, M. Georgiou, R. Haagmans, and K. Tsinganos, ULF wave activity during the 2003 Halloween superstorm: multipoint observations from CHAMP, Cluster and Geotail missions, Ann. Geophys., 30, 1751-1768, doi:10.5194/angeo30-1751-2012, 2012.

Balogh, A. et al., The cluster magnetic field investigation, Space Sci. Rev., 79, 65-92, 1997.

Brautigam, D. H. and J. M. Albert, Radial diffusion analysis of outer radiation belt electrons during the October 9, 1990, magnetic storm, $J$. Geophys. Res., 105(A1), 291-309, doi:10.1029/1999JA900344, 2000.

Chen, L. and A. Hasegawa, Kinetic theory of geomagnetic pulsations: 1. Internal excitations by energetic particles, J. Geophys. Res., 96, 1503$1512,1991$.

Cheng, C. Z. and C. S. Lin, Eigenmode analysis of compressional waves in the magnetosphere, Geophys. Res. Lett., 14, 884-887, 1987.

Chi, P. J. and C. T. Russell, Use of the Wigner-Ville distribution in interpreting and identifying ULF waves in triaxial magnetic records, $J$. Geophys. Res., 113, A01218, 2008.

Constantinescu, O. D., K.-H. Glassmeier, P. M. E. Decreau, M. Franz, and K.-H. Fornacon, Low frequency wave sources in the outer magnetosphere, magnetosheath, and near Earth solar wind, Ann. Geophys., 25, 2217-2228, doi:10.5194/angeo-25-2217-2007, 2007.

Elkington, S. R., A review of ULF interactions with radiation belt electrons, in Magnetospheric ULF Waves: Synthesis and New Directions, edited by K. Takahashi et al., Geophys. Monagr. Ser., 169, 177-193, doi:10.1029/169GM12, 2006.

Engebretson, M. J., J. L. Posch, A. M. Westerman, N. J. Otto, J. A. Slavin, G. Le, R. J. Strangeway, and M. R. Lessard, Temporal and spatial characteristics of Pc1 waves observed by ST5, J. Geophys. Res., 113, A07206, doi:10.1029/2008JA013145, 2008.

Escoubet, C. P., R. Schmidt, and M. L. Goldstein, Cluster-Science and mission overview, Space Sci. Rev., 79, 11-32, 1997.

Friis-Christensen, E., H. Luhr, and G. Hulot, Swarm: A constellation to study the Earth's magnetic field, Earth Planets Space, 58, 351-358, 2006.

Gustafsson, G. et al., The electric field and wave experiment for the Cluster Mission, Space Sci. Rev., 79, 137-156, 1997.

Jacobs, J. A., Y. Kato, S. Matsushita, and V. A. Troitskaya, Classification of geomagnetic micropulsations, J. Geophys. Res., 69, 180-181, 1964.

Han, D. S., T. Iyemori, M. Nose, H. McCreadie, Y. Gao, F. Yang, S. Yamashita, and P. Stauning, A comparative analysis of low-latitude Pi 2 pulsations observed by Oersted and ground stations, J. Geophys. Res., 109, A10209, 2004.

Heilig, B., H. Lühr, and M. Rother, Comprehensive study of ULF upstream waves observed in the topside ionosphere by CHAMP and on the ground, Ann. Geophys., 25, 737-754, 2007.

Kataoka, R., Y. Miyoshi, and A. Morioka, Hilbert-Huang Transform of geomagnetic pulsations at auroral expansion onset, J. Geophys. Res., 114, A09202, 2009.

Kim, K.-H. and K. Takahashi, Statistical analysis of compressional Pc34 pulsations observed by AMPTE CCE at $\mathrm{L}=2-3$ in the day-side magnetosphere, J. Geophys. Res., 104, 4539-4558, 1999.

Kunagu, P., G. Balasis, V. Lesur, and E. Chandrasekhar, Wavelet characterization of external magnetic sources as observed by CHAMP satellite: evidence for unmodeled signals in geomagnetic field models, Geophys. J. Int., 192, 946-950, doi:10.1093/gji/ggs093, 2013.

Le, G., P. J. Chi, R. J. Strangeway, and J. A. Slavin, Observations of a unique type of ULF wave by low altitude Space Technology 5 satellites, J. Geophys. Res., 116, A08203, 2011.

Lee, E. A., I. R. Mann, T. M. Loto'aniu, and Z. C. Dent, Global Pc5 pulsations observed at unusually low L during the great magnetic storm of 24 March 1991, J. Geophys. Res., 112, A05208, 2007.

Macmillan, S. and S. Maus, International Geomagnetic Reference FieldThe tenth generation, Earth Planets Space, 57(12), 1135-1140, 2005. 
Mann, I. R., D. K. Milling, I. J. Rae, L. G. Ozeke, A. Kale, Z. C. Kale, K. R. Murphy, A. Parent, M. Usanova, D. M. Pahud, E.-A. Lee, V. Amalraj, D. D. Wallis, V. Angelopoulos, K.-H. Glassmeier, C. T. Russell, H.-U. Auster, and H. J. Singer, The upgraded CARISMA magnetometer array in the THEMIS era, Space Sci. Rev., 141, 413-451, 2008.

Maus, S., M. Rother, K. Hemant, C. Stolle, H. L̈̈r, A. Kuvshinov, and N. Olsen, Earth's lithospheric magnetic field determined to spherical harmonic degree 90 from CHAMP satellite measurements, Geophys. $J$. Int., 164, 319-330, 2006.

Menk, F., Magnetospheric ULF waves: A review, in The Dynamic Magnetosphere, edited by Liu, W. and M. Fujimoto, IAGA Special Sopron Book Series, Springer, 3, 223, 2011.

Ndiitwani, D. C. and P. R. Sutcliffe, The structure of low-latitude Pc3 pulsations observed by CHAMP and on the ground, Ann. Geophys., 27, 1267-1277, 2009.

Ozeke, L. G., I. R. Mann, K. R. Murphy, I. J. Rae, D. K. Milling, S. R. Elkington, A. A. Chan, and H. J. Singer, ULF wave derived radiation belt radial diffusion coefficients, J. Geophys. Res., 117, A04222, doi:10.1029/2011JA017463, 2012.

Pickett, J. S., B. Grison, Y. Omura, M. J. Engebretson, I. Dandouras, A. Masson, M. L. Adrian, O. Santolik, P. M. E. Decreau, N. CornilleauWehrlin, and D. Constantinescu, Cluster observations of EMIC triggered emissions in association with Pc1 waves near Earth's plasmapause, Geophys. Res. Lett., 37, L09104, doi:10.1029/2010GL042648, 2010.

Pilipenko, V., E. Fedorov, B. Heilig, M. J. Engebretson, P. Sutcliffe, and H. Lühr, ULF waves in the topside ionosphere: Satellite observations and modeling, in The Dynamic Magnetosphere, IAGA Special Sopron Book Series 3, doi:10.1007/978-94-007-0501-2_14, Springer, 2011.

Reigber, C., H. Lühr, and P. Schwintzer, CHAMP Mission Status, Adv. Space Res., 30, 129-134, 2002.

Samson, J. C., Descriptions of the polarization states of vector processes: Applications to ULF magnetic fields, Geophys. J. R. Astron. Soc., 34, 403-419, 1973.

Santolik, O., M. Parrot, and F. Lefeuvre, Singular value decomposition methods for wave propagation analysis, Radio Sci., 38, 1010, doi:10.1029/2000RS002523, 2003.

Sarris, T. E., W. Liu, K. Kabin, X. Li, S. R. Elkington, R. Ergun, R. Rankin, V. Angelopoulos, J. Bonnell, K. H. Glassmeier, and U. Auster,
Characterization of ULF pulsations by THEMIS, Geophys. Res. Lett., 36, L04104, doi:10.1029/2008GL036732, 2009.

Slavin, J. A., G. Le, R. J. Strangeway, Y. Wang, S. A. Boardsen, M. B. Moldwin, and H. E. Spence, Space technology 5 multi-point measurements of near-Earth magnetic fields: Initial results, Geophys. Res. Lett., 35, L02107, doi:10.1029/2007GL031728, 2008.

Stolle, C., H. Luhr, M. Rother, and G. Balasis, Magnetic signatures of equatorial spread $\mathrm{F}$ as observed by the CHAMP satellite, J. Geophys. Res., 111, A02304, doi:10.1029/2005JA011184, 2006.

Sutcliffe, P. R. and H. Lühr, A comparison of Pi2 pulsations observed by CHAMP in low Earth orbit and on the ground at low latitudes, Geophys. Res. Lett., 30, 2105, doi:10.1029/2003GL018270, 2003.

Sutcliffe, P. R. and H. Lühr, A search for dayside geomagnetic Pi2 pulsations in the CHAMP low-Earth-orbit data, J. Geophys. Res., 115, A05205, doi:10.1029/2009JA014757, 2010.

Takahashi, K. and B. Anderson, Distribution of ULF energy ( $\mathrm{f} \leq 80 \mathrm{mHz})$ in the inner magnetosphere: A statistical analysis of AMPTE CCE magnetic field data, J. Geophys. Res., 97, 10,751, 1992.

Takahashi, K., B. J. Anderson, and R. J. Strangeway, AMPTE CCE observations of Pc 3-4 Pulsations at L = 2-6, J. Geophys. Res., 95, 1717917186, 1990.

Teramoto, M., K. Takahashi, M. Nose, D.-H. Lee, and P. R. Sutcliffe, Pi2 pulsations in the inner magnetosphere simultaneously observed by the Active Magnetospheric Particle Tracer Explorers/Charge Composition Explorer and Dynamics Explorer 1 satellites, J. Geophys. Res., 116, doi:10.1029/2010JA016199, 2011.

Usanova, M. E., I. R. Mann, I. J. Rae, Z. C. Kale, V. Angelopoulos, J. W. Bonnell, K.-H. Glassmeier, H. U. Auster, and H. J. Singer, Multipoint observations of magnetospheric compressionrelated EMIC Pc1 waves by THEMIS and CARISMA, Geophys. Res. Lett., 35, L17S25, doi:10.1029/2008GL034458, 2008.

Vellante, M. et al., Ground/satellite signatures of field line resonance: A test of theoretical predictions, J. Geophys. Res., 109, A06210, doi:10.1029/2004JA010392, 2004.

G. Balasis (e-mail: gbalasis@noa.gr), I. A. Daglis, M. Georgiou, C. Papadimitriou, and R. Haagmans 